\title{
Intruder Detection System using Posture Recognition and Machine Learning
}

\author{
Mainak Bhattacharya Shiladitya Pujari, PhD \\ Department of \\ Information Technology \\ University Institute of \\ Technology \\ The University of \\ Burdwan
}

\author{
Ankit Anand \\ Department of \\ Information Technology \\ University Institute of \\ Technology \\ The University of \\ Burdwan
}

\author{
Niranjan Kumar \\ Department of \\ Information \\ Technology \\ University Institute of \\ Technology \\ The University of \\ Burdwan
}

\author{
Sumit Kumar Jha \\ Department of Information \\ Technology \\ University Institute of \\ Technology
}

\author{
Aryan Raj \\ Department of Information \\ Technology \\ University Institute of \\ Technology
}

\author{
Sk Masum Hossain \\ Department of Information \\ Technology \\ University Institute of \\ Technology
}

\begin{abstract}
Generally, Science and technology always being persuaded when there is a need. Being secured is the utmost desire of every creatures in this world of insecurity. It is very much tough to have human security systems everywhere all the time. So in that case a system capable of atleast detection and warning of possible threats or dangers can be welcomed. In this context, one of the best way to achieve this goal is to build an intruder detection system. Intruders may be physical in nature or in case of computer networks there are network intruders also. But here in this paper it has been tried to present a comprehensive study about physical intruder detection system. In this attempt it has been presented a detailed hypothesis about intruder detection system in the light of gait recognition. It is also put in front of how research works had been done in earlier days and also used some machine learning algorithms on some human gait datasets to obtain results which is thought to give a brief idea in this domain.
\end{abstract}

\section{Keywords}

Posture Recognition, Intruder, Gait recognition

\section{INTRODUCTION}

Biometric authentication is a process where a person is identified and verified considering his or her physical characteristics which can be fingerprints, iriscan, facescan, gait recognition. Depending on the desired security single/multiple authentication characteristics can be used. In this paper a deep focus has been made on gait recognition based authentication. This is an authentication process where a person's walking style is depicted. Early studies noted that 24 different parts of human gait can be used to identify a person . It has been also seen in the early studies that light points attached to individuals joint positions used to determine human motion activities. Many researches showed that each individual had unique body structure which specifies him accordingly. Hence gait recognition is topic of high interest in biometric authentication research community. With the development of variety of cameras and mobile phones with built-in sensors such as accelerometers and gyroscopes have added a new taste in gait based authentication. So in this paper a brief analysis has been given on how gait recognition can be done and how much it is useful in the coming days.

\section{POSTURE RECOGNITION}

Human posture recognition is an alluring and demanding topic in machine learning due to its outstanding applications of environmental awareness, social awareness, surveillance systems .In difficult working environment posture recognition paves the way for interaction between human and computer rather than the traditional ones with improved accuracies and efficiency. Existing techniques which foregoes in both specific direction and walk scenes, posture recognition compared to these performs far better involving standing, sitting, lying, bending, running of individuals . This intimates us a minute change in posture or movements of an individual is sufficient enough to identify that very person .[3]

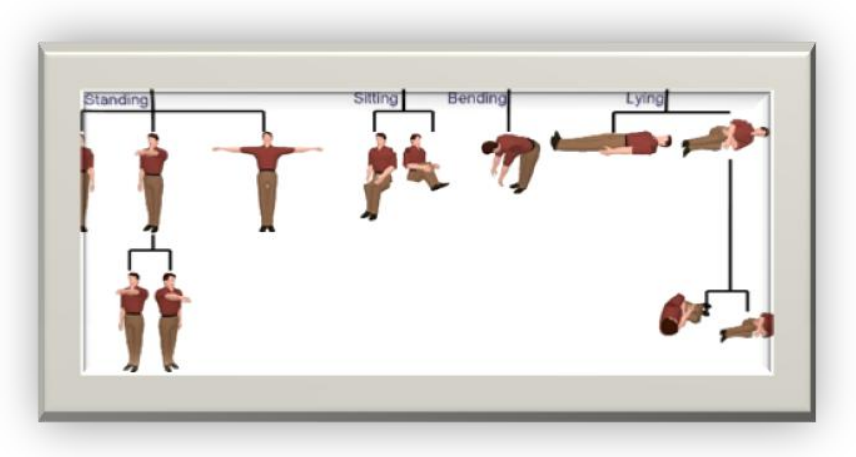




\section{CHARACTERISTICS OF GAIT RECOGNITION}

Gait recognition has several properties which are unique in nature:-

Firstly,Gait based authentication does not require direct physical contact with the person whereas other authentication techniques require physical contact. Secondly gait recognition mainly includes human motion so, if someone tries to hide his or her face it will not affect the results. Thirdly human gait recognition system is independent of distance..Fourthly, gait recognition can also be done using low resolution cameras which is cost effective.. Fifthly, gait recognition can also be done with the help of camera, an accelerometer present in a smartphone ,a floor sensor, or even a radar could be used for collecting human gait data. Sixthly, gait features due to its uniqueness are very difficult to be inculcated as it uses human activities and silhouettes. This property is very useful in case of crime analysis.[7-10].

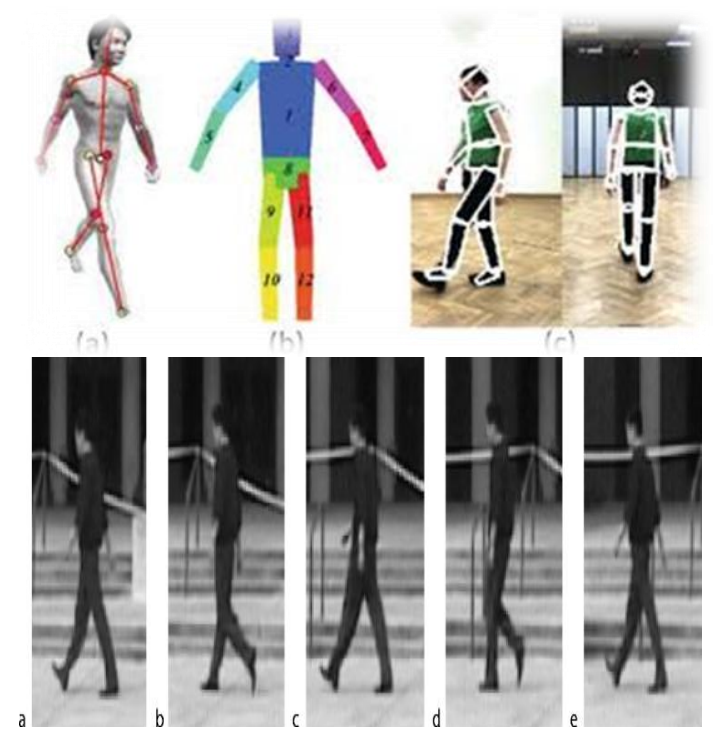

\section{RELATED WORK}

Various posture recognition methods gives the discernible information of human being and 2 dimensional posture data by performing feature extraction from their images. Ramanan and Sminchisescu enlighted an algorithm using Gradient Descent Method to predict human postures using human outline/contour traces to compare and contrast with human edge standards . Souto and Musse with the help of artificial neural networks detected human poses in a single image automatically. To derive human skeletal structure using features of static images, this procedure incurres lot of cumbersome computation to extract features . skeletal joints angle to depict the human postures and a multi-level support vector machine (SVM) and a decision forest were used in past by Miranda et.al to classify.It does not perform well when multiple similar postures are in the business. Agarwal and Triggs elaborated about a relevance vector machine (RVM) based regression method that employs outline specification to depict human postures. Zainordin et al. persuaded a method to classify postures with the help of threshold distance, joint angles ,skeleton and depth information. Hoppe et al. argumented about an intrusion detection method by analysing attack patterns which is already defined in the database Larson et al. proposed a specification-based approach, comparing and contrasting the appearance of the current specification system to the designated patterns. [1, 2, 4, 5, 6, 27, 36]

\section{FRAMEWORK}

A typical gait recognition system has four phases, namely Data Acquisition, Feature Representation, Dimension Reduction, And Classification.

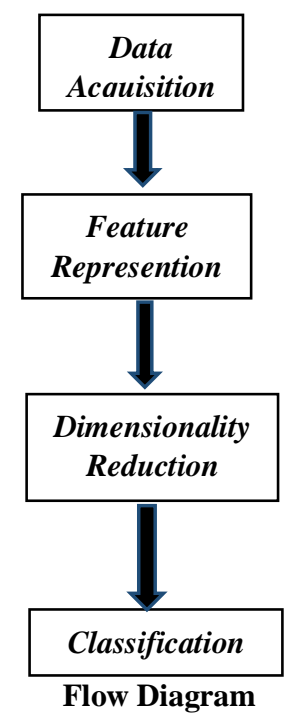

\subsection{Data Acquisition}

In this step human gait data is being collected. Human gait data can be said as a collection of spatio- temporal data. Human gait data consists of human motion and activities. This human gait data can be collected with the help of different types of sensors such as cameras, floor sensors, accelerometers, radars etc .

Camera : Each and every person have their own style of walking. If we are able to capture the gait images properly, by analysing them we can identify that person. In this case camera as a data collection device is much helpful. Inspite of placing the camera far away from the individual it is very much successful to collect the images or the videos which can be considered as gait raw data for further processing.

Floor sensor: As mentioned earlier when a person walks his/her walking style is unique compared to others in that manner his/her foot pressure also differs from others. Foot pressure data can be collected with the help of commercial force platforms and Baropodometric mats such as Force platform AMTI series OR6-7 of Biometrics France, Kistler Force plate of different types, Dynamometric mat ADAL of Tecmachine etc.

Accelerometer: When a person is walking his /her acceleration during walking can also be measured. Accelerations of humans in 3D space varies from one individual to other. Similar to camera, foot pressure devices accelerometer also plays pivotal role in gait data acquisition. Piezoresistive and capacitive accelerometers provide dual acceleration components with higher stability. Hence these are suitable to measure the motion status in the human gait.

Radar: During walking each and every individual generate different doppler signatures . If it can be captured it eases the 
procedure of gait recognition . Radar, specifically continuous wave radar gives the opportunity to collect gait data being distant from the individual. Continuous wave radar optimizes the use of dielectric resonant oscillator for generating signals and for transmitting and receiving signal purposes two different antennas are being used .[11,13,15]

\subsection{Feature Representation}

In the purpose of recognizing individuals, raw data which are collected during the data acquisition phase, gait features must be extracted from them by the gait recognition system. In the feature representation step the unprocessed data is transformed into some specific patterns. Mainly if we talk about video based gait recognition, the types of feature representation techniques boils down to two major threads one is model based and another is model-free methods . Some researches showed as an example that model based feature representation method can be performed by dividing human silhouettes into 7 constituent parts, each part was fitted in an elliptical structure and different parameters of those structures were counted as features .
(a) Model-based Feature Representation method for Video Data
In this specific feature representation method whole human body is modelled and features are being extracted from this model . It does not depend on scale and view. But it is upto some extent dependent on the quality of the video. After modelling the entire human body, model-based feature representation methods mainly use distances or angles between the joints on human body for recognition.

\section{(b) Model-free Feature Representation method for Video Data}

Compare to Model based feature representation method Model-free feature representation method mainly focuses to forego its proceedings with the entire motion or shape and size of human silhouettes. Model-free feature representation is advantageous because whether the video quality is good or bad its performance remains unaffected and paves the way to be instantiated distant from the human . It is very much cost effective . When data captured from different viewpoints, viewing angles change significantly as model free feature representation method is dependant on scale and viewpoint in this case recognition rate faces a downturn .[12,14,16,20,22]

\subsection{Dimensionality reduction}

Real life datasets actually is very much huge in size and quantity . Deriving any certain patterns from these datasets is pretty much difficult to perform and classification results which will come from this may not reach satisfactory level so it is very much ardent need to reduce the dimensionality of the data. Some features may not be important for analysis purposes so better to deduct them. In this step of Dimensionality Reduction non important and bad quality features are being excluded for further considerations. It can be broadly classified into two types, namely outliers removal and feature reduction.

\section{(a) Feature Reduction}

Feature Reduction is a subset of dimensionality reduction which can evade bad quality features and which are not relevant as far as effectivity and efficiency of gait based biometric system is concerned. There are many feature reduction methods, as discussed below.

$>$ Linear Discriminant Analysis (LDA). LDA finds its position in the supervised machine learning feature detection genre. Firstly it computes the within class and between class scatter matrices and after that computing the eigen vectors of them it sorts the eigen values and selects the top $t$ and further creates a new matrix containing eigen vectors $t$ eigen values . At last by taking the dot product of the data and the matrix it obtains new features .

$>$ Principal Component Analysis (PCA). PCA is one of the famous feature reduction procedures in the modern era. It very much works well when a dataset size is huge where reduction of the dimensionality is a challenge with increasing interpretability and also at the same time it gives the assurance that information loss is bare minimum .

$>$ Piecewise Linear Representation (PLR). PLR is a simple approach to be considered for feature reduction. It rejects a small portion of the signal which are deviating from the standard and keeps the majority of time series signals on signals.

$>$ I-vector. I-vector is one of the well organized feature reduction approaches for gait recognition systems. Generally, the i-vector method takes Gaussian Mixture Model with Universal Background Model for building class dependent model .

$>$ Discrete Cosine Transform (DCT). Discrete Cosine Transform is very much potent to reduce the dimensionality of features . Basically it truncates the DCT components which have high frequency value and leftover components are used for further classification . [17,21,25]

(b) Outliers Removal

To explain outliers we can say outliers are the abrupt values that digress from other values on the experiment . Outlier removal has find its place in almost all machine learning algorithms. Outliers removal mainly relies on discarding corrupted signals. Some common known outliers in gait based system may be absurd gait sequences, at start walking posture of an individual may not be similar with the ending one, also when people disguise themselves with different types of clothes or carry any objects may be considered as an outlier .

\subsection{Classification}

Classification is a procedure that can be followed to categorize a particular set of data into classes. Though it can be persuaded on both structured data and unstructured data. The method initiates with predicting the class of datapoints and the here the classes are often being referred to as label or target. Predictive modelling classification approach somehow approximates the mapping function from input data variables to output data variables which are discrete in nature. Main focus is to determine in which class the data (new) will lie. Here we are trying to make attention some of those well known classification methodologies.

\section{* Machine Learning}

Machine learning is a paradigm that talks about to learn and make predictions depending on the 
acquired data. The working procedure of ML algorithms is by building a model to continue its data based predictions and decisions taken from gathered data . It is a field of study that give the conclusions from the already acquired data by performing statistical and mathematical operations and makes the assumptions about what is not known with those conclusions. When the question come about gait biometric system ML based classification generally is a kind of supervised learning which gives the notion of procedure of concluding a function with the help of labelled training data by mapping those training data to its specific label .

* Distance

Distance metrics is one of the well known and easy to use classification methods which measures similarity between the acquired data. In distance metrics there are many well known distances named like Euclidean distance , Manhattan distance , Minkowski distance , Hamming distance . In gait based biometric systems Euclidean distance is mostly used . In some works Manhattan distance also used as far as gait recognition is concerned. Euclidean distance is calculated using the following formula :-

$$
D_{E}=\sum_{i=1}^{n}\left|a_{i}-b_{i}\right|^{1 / 2}
$$

In which $D_{\mathrm{E}}$ stands for Euclidean distance between test data and center of the training data.

\section{* Correlation}

The term 'correlation', in this field of study noted as a technique determining relationships between different variables. Pearson's Correlation Coefficient commonly which is known to us , gives the idea in the form of if we want to measure correlation between two datasets $A$ and $B$ where $A=\left\{a_{1}, a_{2}, \ldots \ldots \ldots a_{n}\right\}$ and $B=\left\{b_{1}, b_{2}\right.$, $\left.b_{n}\right\}$ is given by the following equation $[18,19,23,24,26]$.

$$
C(A, B)=\sum_{i=1}^{n} \frac{\left(a_{i}-\bar{a}\right)\left(b_{i}-\bar{b}\right)}{\left[\sum_{i=1}^{n}\left(a_{i}-a\right)^{2} \sum_{i=1}^{n}\left(b_{i}-b\right)^{2}\right]^{1 / 2}}
$$

\section{EXPERIMENT AND RESULT}

Here in this work some publicly available datasets have been used from one of the famous websites Kaggle. The GaitPhase database comprises of gait data from 21 individuals $(10$ male, 11 female, age: $23.8 \mathrm{yrs} \pm 3.3 \mathrm{yrs}$, height: $172.8 \mathrm{~cm} \pm 9.4 \mathrm{~cm}$, weight: $66.6 \mathrm{~kg} \pm 10.9 \mathrm{~kg}$; all values were mean \pm standard deviation). In total, 25306 steps were gathered .The exercise was performed by walking on a split-belt treadmill at 12 different speeds in the interval of $[0.6,1.7] \mathrm{m} / \mathrm{s}$ with $0.1 \mathrm{~m} / \mathrm{s}$ increments for one minute at each speed . 3D kinematic data using a Qualisys (Qualisys AB, Gothenburg, Sweden) motion capture system with 8 Oqus cameras with sampling rate $200 \mathrm{~Hz}$ and an instrumented Bertec (Bertec Corporation, Columbus, OH, USA) split-belt treadmill with built-in force plates with sampling rate 1000
$\mathrm{Hz}$ were used for data acquisition. Both systems were being synchronized according to camera frame-wise .Firstly some publicly available gait datasets were being collected as mentioned above and tested the algorithms and the results were collected. All of these have been performed through WEKA 3.8.5 software, it is a assimilation of machine learning algorithms for data mining tasks. It contains tools for data preprocessing, classification, regression, clustering purposes, association rules mining, and visualization of results. Firstly the datasets were preprocessed with the algorithms and made it ready for further classification . Principal Component Analysis (PCA) and Discrete Wavelet Transform (DWT) have been used as feature reduction techniques and Random Forest as classifier. To check the results in different aspects the cross validation have been varied into $100,1000,10 \mathrm{~s}$ of folds . As numeric datasets were used so in WEKA results were in terms of Correlation Coefficient , Mean absolute error (MAE), Root mean squared error(RMSE), Relative absolute error(RAE), Root relative squared error(RRSE) . Here Correlation Coefficient tells about variance of data explained by the model . Mean absolute error is the average distance between the actual data points and the model predictions. Root mean squared error is a bit different way of calculating mean absolute error . The next two columns i.e RAE and RRSE scale the error to the mean paves the way to compare between models constructed with larger or smaller valued data.

Table 1: Shows results of Gaitphase Datasets after performing Gait Recognition Algorithms in Weka

\begin{tabular}{|c|c|c|c|c|c|c|c|}
\hline $\begin{array}{l}\text { Dat } \\
\text { aset }\end{array}$ & $\begin{array}{l}\text { Algor } \\
\text { ithm }\end{array}$ & $\begin{array}{l}\text { Fo } \\
\text { ld }\end{array}$ & $\begin{array}{l}\text { Correl } \\
\text { ation- } \\
\text { Coeffi } \\
\text { cient }\end{array}$ & $\begin{array}{l}\text { Ma } \\
\text { e }\end{array}$ & $\begin{array}{l}\mathbf{R m} \\
\text { se }\end{array}$ & Rae & Rrse \\
\hline 1 & $\begin{array}{l}\text { PCA- } \\
\text { RF }\end{array}$ & $\begin{array}{l}10 \\
0\end{array}$ & 0.9995 & $\begin{array}{l}0.00 \\
06\end{array}$ & $\begin{array}{l}0.00 \\
08\end{array}$ & $\begin{array}{l}2.86 \\
38 \%\end{array}$ & $\begin{array}{l}3.28 \\
33 \%\end{array}$ \\
\hline 1 & $\begin{array}{l}\text { PCA- } \\
\text { RF }\end{array}$ & $\begin{array}{l}10 \\
00\end{array}$ & 0.9995 & $\begin{array}{l}0.00 \\
06\end{array}$ & $\begin{array}{l}0.00 \\
08\end{array}$ & $\begin{array}{l}2.86 \\
43 \%\end{array}$ & $\begin{array}{l}3.29 \\
76 \%\end{array}$ \\
\hline 1 & $\begin{array}{l}\text { DWT } \\
\text {-RF }\end{array}$ & $\begin{array}{l}10 \\
0 \\
\end{array}$ & 0.9995 & $\begin{array}{l}0.00 \\
06 \\
\end{array}$ & $\begin{array}{l}0.00 \\
08 \\
\end{array}$ & $\begin{array}{l}2.86 \\
38 \%\end{array}$ & $\begin{array}{l}3.28 \\
33 \%\end{array}$ \\
\hline 1 & $\begin{array}{l}\text { DWT } \\
\text {-RF }\end{array}$ & $\begin{array}{l}10 \\
00\end{array}$ & 0.9996 & $\begin{array}{l}0.00 \\
05\end{array}$ & $\begin{array}{l}0.00 \\
07\end{array}$ & $\begin{array}{l}2.50 \\
8 \%\end{array}$ & $\begin{array}{l}2.81 \\
94 \% \\
\end{array}$ \\
\hline 2 & $\begin{array}{l}\text { PCA- } \\
\text { RF }\end{array}$ & $\begin{array}{l}10 \\
0\end{array}$ & 0.9991 & $\begin{array}{l}9.60 \\
6\end{array}$ & $\begin{array}{l}15.3 \\
786\end{array}$ & $\begin{array}{l}2.71 \\
19 \%\end{array}$ & $\begin{array}{l}4.14 \\
52 \%\end{array}$ \\
\hline 2 & $\begin{array}{l}\text { PCA- } \\
\text { RF }\end{array}$ & $\begin{array}{l}10 \\
00\end{array}$ & 0.9991 & $\begin{array}{l}9.58 \\
61\end{array}$ & $\begin{array}{l}15.3 \\
439\end{array}$ & $\begin{array}{l}2.70 \\
63 \%\end{array}$ & $\begin{array}{l}4.13 \\
59 \%\end{array}$ \\
\hline 2 & $\begin{array}{l}\text { DWT } \\
\text {-RF }\end{array}$ & $\begin{array}{l}10 \\
0 \\
\end{array}$ & 0.9996 & $\begin{array}{l}6.82 \\
06 \\
\end{array}$ & $\begin{array}{l}10.1 \\
122 \\
\end{array}$ & $\begin{array}{l}1.92 \\
56 \% \\
\end{array}$ & $\begin{array}{l}2.72 \\
57 \% \\
\end{array}$ \\
\hline 2 & $\begin{array}{l}\text { DWT } \\
\text {-RF }\end{array}$ & $\begin{array}{l}10 \\
00\end{array}$ & 0.9997 & $\begin{array}{l}6.45 \\
25\end{array}$ & $\begin{array}{l}9.69 \\
6\end{array}$ & $\begin{array}{l}1.82 \\
17 \%\end{array}$ & $\begin{array}{l}2.61 \\
35 \%\end{array}$ \\
\hline 3 & $\begin{array}{l}\text { PCA- } \\
\text { RF }\end{array}$ & $\begin{array}{l}10 \\
0\end{array}$ & 0.9992 & $\begin{array}{l}0.00 \\
08\end{array}$ & $\begin{array}{l}0.00 \\
01\end{array}$ & $\begin{array}{l}3.45 \\
87 \%\end{array}$ & $\begin{array}{l}3.99 \\
34 \%\end{array}$ \\
\hline 3 & $\begin{array}{l}\text { PCA- } \\
\text { RF }\end{array}$ & $\begin{array}{l}10 \\
00\end{array}$ & 0.9992 & $\begin{array}{l}0.00 \\
07\end{array}$ & $\begin{array}{l}0.00 \\
1\end{array}$ & $\begin{array}{l}3.44 \\
56 \%\end{array}$ & $\begin{array}{l}3.95 \\
14 \%\end{array}$ \\
\hline 3 & $\begin{array}{l}\text { DWT } \\
\text {-RF }\end{array}$ & $\begin{array}{l}10 \\
0\end{array}$ & 0.9996 & $\begin{array}{l}0.00 \\
05\end{array}$ & $\begin{array}{l}0.00 \\
07\end{array}$ & $\begin{array}{l}2.40 \\
5 \%\end{array}$ & $\begin{array}{l}2.76 \\
19 \%\end{array}$ \\
\hline 3 & $\begin{array}{l}\text { DWT } \\
\text {-RF }\end{array}$ & $\begin{array}{l}10 \\
00\end{array}$ & 0.9996 & $\begin{array}{l}0.00 \\
05\end{array}$ & $\begin{array}{l}0.00 \\
07\end{array}$ & $\begin{array}{l}2.39 \\
78 \%\end{array}$ & $\begin{array}{l}2.74 \\
44 \%\end{array}$ \\
\hline 4 & $\begin{array}{l}\text { PCA- } \\
\text { RF }\end{array}$ & $\begin{array}{l}10 \\
0\end{array}$ & 0.9993 & $\begin{array}{l}9.48 \\
6\end{array}$ & $\begin{array}{l}14.3 \\
555\end{array}$ & $\begin{array}{l}2.76 \\
27 \%\end{array}$ & $\begin{array}{l}3.85 \\
06 \%\end{array}$ \\
\hline 4 & $\begin{array}{l}\text { PCA- } \\
\text { RF }\end{array}$ & $\begin{array}{l}10 \\
00\end{array}$ & 0.9993 & $\begin{array}{l}9.47 \\
94\end{array}$ & $\begin{array}{l}14.3 \\
464\end{array}$ & $\begin{array}{l}2.67 \\
08 \%\end{array}$ & $\begin{array}{l}3.84 \\
83 \%\end{array}$ \\
\hline 4 & $\begin{array}{l}\text { DWT } \\
\text {-RF }\end{array}$ & $\begin{array}{l}10 \\
0\end{array}$ & 0.9996 & $\begin{array}{l}7.13 \\
24\end{array}$ & $\begin{array}{l}10.3 \\
839\end{array}$ & $\begin{array}{l}2.00 \\
96 \%\end{array}$ & $\begin{array}{l}2.78 \\
54 \%\end{array}$ \\
\hline 4 & DWT & 10 & 0.9996 & 7.12 & 10.3 & 2.00 & 2.78 \\
\hline
\end{tabular}
Software 


\begin{tabular}{|c|c|c|c|c|c|c|c|}
\hline & -RF & 00 & & 92 & 787 & $87 \%$ & $4 \%$ \\
\hline \multirow[t]{2}{*}{5} & PCA- & 10 & 0.9989 & 0.00 & 0.00 & 3.93 & 4.60 \\
\hline & RF & 0 & & 09 & 12 & $44 \%$ & $74 \%$ \\
\hline \multirow[t]{2}{*}{5} & PCA- & 10 & 0.999 & 0.00 & 0.00 & 3.93 & 4.59 \\
\hline & RF & 00 & & 09 & 12 & $97 \%$ & $64 \%$ \\
\hline \multirow[t]{2}{*}{5} & DWT & 10 & 0.9997 & 0.00 & 0.00 & 2.36 & 2.64 \\
\hline & $-R F$ & 0 & & 05 & 07 & $07 \%$ & $01 \%$ \\
\hline \multirow[t]{2}{*}{5} & DWT & 10 & 0.999 & 7.77 & 12.2 & 3.45 & 4.63 \\
\hline & $-R F$ & 00 & & 65 & 332 & $21 \%$ & $59 \%$ \\
\hline \multirow[t]{2}{*}{6} & PCA- & 10 & 0.9997 & 3.45 & 4.97 & 1.65 & 2.25 \\
\hline & $\mathrm{RF}$ & 0 & & 51 & 91 & $33 \%$ & $67 \%$ \\
\hline \multirow[t]{2}{*}{6} & PCA- & 10 & 0.9997 & 3.44 & 4.96 & 1.64 & 2.24 \\
\hline & RF & 00 & & 16 & 32 & $68 \%$ & $94 \%$ \\
\hline \multirow[t]{2}{*}{6} & DWT & 10 & 0.9997 & 3.45 & 4.97 & 1.65 & 2.25 \\
\hline & $-R F$ & 0 & & 51 & 91 & $33 \%$ & $67 \%$ \\
\hline \multirow[t]{2}{*}{6} & DWT & 10 & 0.9997 & 3.44 & 4.96 & 1.65 & 2.25 \\
\hline & $-R F$ & 00 & & 87 & 91 & $02 \%$ & $21 \%$ \\
\hline \multirow[t]{2}{*}{7} & PCA- & 10 & 0.999 & 0.00 & 0.00 & 3.86 & 4.48 \\
\hline & RF & 0 & & 07 & 09 & $39 \%$ & $7 \%$ \\
\hline \multirow[t]{2}{*}{7} & PCA- & 10 & 0.9992 & 3.00 & 0.00 & 3.33 & 3.99 \\
\hline & $\mathrm{RF}$ & 00 & & 06 & 08 & $02 \%$ & $63 \%$ \\
\hline \multirow[t]{2}{*}{7} & DWT & 10 & 0.9994 & 0.00 & 0.00 & 2.77 & 3.37 \\
\hline & $-R F$ & 0 & & 05 & 07 & $31 \%$ & $66 \%$ \\
\hline \multirow[t]{2}{*}{7} & DWT & 10 & 0.9994 & 0.00 & 0.00 & 2.76 & 3.36 \\
\hline & $-R F$ & 00 & & 05 & 07 & $68 \%$ & $93 \%$ \\
\hline \multirow[t]{2}{*}{8} & PCA- & 10 & 0.9987 & 0.00 & 0.00 & 4.30 & 5.09 \\
\hline & RF & 0 & & 05 & 11 & $69 \%$ & $75 \%$ \\
\hline 8 & PCA- & 10 & 0.9988 & 0.00 & 0.00 & 4.28 & 5.08 \\
\hline & $\mathrm{RF}$ & 00 & & 08 & 11 & $46 \%$ & $02 \%$ \\
\hline 8 & DWT & 10 & 0.9995 & 0.00 & 0.00 & 2.58 & 3.03 \\
\hline & $-R F$ & 0 & & 05 & 07 & $48 \%$ & $89 \%$ \\
\hline 8 & DWT & 10 & 0.9993 & 0.00 & 0.00 & 3.14 & 3.71 \\
\hline & -RF & 00 & & 06 & 08 & $75 \%$ & $83 \%$ \\
\hline 9 & PCA- & 10 & 0.9993 & 9.48 & 14.3 & 2.67 & 3.85 \\
\hline & $\mathrm{RF}$ & 0 & & 6 & 55 & $27 \%$ & $06 \%$ \\
\hline 9 & PCA- & 10 & 0.9993 & 9.47 & 14.3 & 2.67 & 3.83 \\
\hline & $\mathrm{RF}$ & 00 & & 94 & 464 & $08 \%$ & $84 \%$ \\
\hline 9 & DWT & 10 & 0.9996 & 7.13 & 10.3 & 2.00 & 2.78 \\
\hline & $-R F$ & 0 & & 24 & 839 & $96 \%$ & $54 \%$ \\
\hline 9 & DWT & 10 & 0.9996 & 7.12 & 10.3 & 2.00 & 2.78 \\
\hline & -RF & 00 & & 92 & 787 & $87 \%$ & $4 \%$ \\
\hline 10 & PCA- & 10 & 0.998 & 0.00 & 0.00 & 5.22 & 6.31 \\
\hline & $\mathrm{RF}$ & 0 & & 1 & 14 & $2 \%$ & $27 \%$ \\
\hline 10 & PCA- & 10 & 0.9981 & 0.00 & 0.00 & 5.21 & 6.29 \\
\hline & RF & 00 & & 1 & 14 & $1 \%$ & $75 \%$ \\
\hline 10 & DWT & 10 & 0.9995 & 0.00 & 0.00 & 2.71 & 3.21 \\
\hline & -RF & 0 & & 05 & 07 & $28 \%$ & $87 \%$ \\
\hline 10 & DWT & 10 & 0.9995 & 0.00 & 0.00 & 2.71 & 3.21 \\
\hline & $-R F$ & 00 & & 05 & 07 & $97 \%$ & $56 \%$ \\
\hline 11 & PCA- & 10 & 0.9988 & 0.00 & 0.00 & 3.95 & 4.86 \\
\hline & RF & 0 & & 06 & 09 & $99 \%$ & $11 \%$ \\
\hline 11 & PCA- & 10 & 0.9989 & 0.00 & 0.00 & 3.92 & 4.81 \\
\hline & RF & 00 & & 06 & 09 & $32 \%$ & $55 \%$ \\
\hline 11 & DWT & 10 & 0.999 & 0.00 & 0.00 & 3.91 & 4.82 \\
\hline & -RF & 0 & & 05 & 08 & $72 \%$ & $35 \%$ \\
\hline 11 & DWT & 10 & 0.9987 & 0.00 & 0.00 & 3.93 & 4.84 \\
\hline & $-R F$ & 00 & & 06 & 08 & $27 \%$ & $23 \%$ \\
\hline 12 & PCA- & 10 & 0.9987 & 0.00 & 0.00 & 4.33 & 5.15 \\
\hline & RF & 0 & & 9 & 12 & $82 \%$ & $94 \%$ \\
\hline 12 & PCA- & 10 & 0.9989 & 0.00 & 0.00 & 4.32 & 5.26 \\
\hline & $\mathrm{RF}$ & 00 & & 9 & 11 & $66 \%$ & $53 \%$ \\
\hline 12 & DWT & 10 & 0.9992 & 0.00 & 0.00 & 3.52 & 3.99 \\
\hline & -RF & 0 & & 07 & 12 & $28 \%$ & $6 \%$ \\
\hline 12 & DWT & 10 & 0.9992 & 0.00 & 0.00 & 3.56 & 3.99 \\
\hline & $-R F$ & 00 & & 08 & 21 & $69 \%$ & $67 \%$ \\
\hline
\end{tabular}

\begin{tabular}{|c|c|c|c|c|c|c|c|}
\hline 13 & $\begin{array}{l}\text { PCA- } \\
\text { RF }\end{array}$ & $\begin{array}{l}10 \\
0\end{array}$ & 0.9992 & $\begin{array}{l}0.00 \\
06\end{array}$ & $\begin{array}{l}0.00 \\
09\end{array}$ & $\begin{array}{l}3.54 \\
49 \%\end{array}$ & $\begin{array}{l}4.00 \\
38 \%\end{array}$ \\
\hline 13 & $\begin{array}{l}\text { PCA- } \\
\text { RF }\end{array}$ & $\begin{array}{l}10 \\
00\end{array}$ & 0.999 & $\begin{array}{l}0.00 \\
05\end{array}$ & $\begin{array}{l}0.00 \\
09\end{array}$ & $\begin{array}{l}3.52 \\
21 \%\end{array}$ & $\begin{array}{l}4.36 \\
56 \%\end{array}$ \\
\hline 13 & $\begin{array}{l}\text { DWT } \\
\text {-RF }\end{array}$ & $\begin{array}{l}10 \\
0\end{array}$ & 0.9992 & $\begin{array}{l}0.00 \\
06\end{array}$ & $\begin{array}{l}0.00 \\
09\end{array}$ & $\begin{array}{l}3.44 \\
52 \%\end{array}$ & $\begin{array}{l}4.00 \\
05 \%\end{array}$ \\
\hline 13 & $\begin{array}{l}\text { DWT } \\
\text {-RF }\end{array}$ & $\begin{array}{l}10 \\
00\end{array}$ & 0.9988 & $\begin{array}{l}0.00 \\
06\end{array}$ & $\begin{array}{l}0.00 \\
07\end{array}$ & $\begin{array}{l}3.92 \\
23 \%\end{array}$ & $\begin{array}{l}4.50 \\
23 \%\end{array}$ \\
\hline 14 & $\begin{array}{l}\text { PCA- } \\
\text { RF }\end{array}$ & $\begin{array}{l}10 \\
0\end{array}$ & 0.9987 & $\begin{array}{l}8.24 \\
53\end{array}$ & $\begin{array}{l}13.2 \\
171\end{array}$ & $\begin{array}{l}3.30 \\
89 \%\end{array}$ & $\begin{array}{l}5.10 \\
86 \%\end{array}$ \\
\hline 14 & $\begin{array}{l}\text { PCA- } \\
\text { RF }\end{array}$ & $\begin{array}{l}10 \\
00\end{array}$ & 0.9987 & $\begin{array}{l}8.23 \\
1\end{array}$ & $\begin{array}{l}13.1 \\
972\end{array}$ & $\begin{array}{l}3.30 \\
31 \%\end{array}$ & $\begin{array}{l}5.10 \\
09 \%\end{array}$ \\
\hline 14 & $\begin{array}{l}\text { DWT } \\
\text {-RF }\end{array}$ & $\begin{array}{l}10 \\
0\end{array}$ & 0.9994 & $\begin{array}{l}5.44 \\
53\end{array}$ & $\begin{array}{l}8.60 \\
22\end{array}$ & $\begin{array}{l}2.18 \\
52 \%\end{array}$ & $\begin{array}{l}3.32 \\
49 \%\end{array}$ \\
\hline 14 & $\begin{array}{l}\text { DWT } \\
\text {-RF }\end{array}$ & $\begin{array}{l}10 \\
00\end{array}$ & 0.9994 & $\begin{array}{l}5.42 \\
64\end{array}$ & $\begin{array}{l}8.58 \\
06\end{array}$ & $\begin{array}{l}2.17 \\
76 \%\end{array}$ & $\begin{array}{l}3.31 \\
65 \%\end{array}$ \\
\hline 15 & $\begin{array}{l}\text { PCA- } \\
\text { RF }\end{array}$ & $\begin{array}{l}10 \\
0\end{array}$ & 0.999 & $\begin{array}{l}0.00 \\
09\end{array}$ & $\begin{array}{l}0.00 \\
12\end{array}$ & $\begin{array}{l}3.94 \\
14 \%\end{array}$ & $\begin{array}{l}4.40 \\
0 \%\end{array}$ \\
\hline 15 & $\begin{array}{l}\text { PCA- } \\
\text { RF }\end{array}$ & $\begin{array}{l}10 \\
00\end{array}$ & 0.9991 & $\begin{array}{l}0.00 \\
09\end{array}$ & $\begin{array}{l}0.00 \\
12\end{array}$ & $\begin{array}{l}3.94 \\
37 \%\end{array}$ & $\begin{array}{l}4.39 \\
14 \%\end{array}$ \\
\hline 15 & $\begin{array}{l}\text { DWT } \\
\text {-RF }\end{array}$ & $\begin{array}{l}10 \\
0\end{array}$ & 0.9996 & $\begin{array}{l}0.00 \\
06\end{array}$ & $\begin{array}{l}0.00 \\
08\end{array}$ & $\begin{array}{l}2.62 \\
76 \%\end{array}$ & $\begin{array}{l}2.96 \\
72 \%\end{array}$ \\
\hline 15 & $\begin{array}{l}\text { DWT } \\
\text {-RF }\end{array}$ & $\begin{array}{l}10 \\
00\end{array}$ & 0.9996 & $\begin{array}{l}0.00 \\
06\end{array}$ & $\begin{array}{l}0.00 \\
08\end{array}$ & $\begin{array}{l}2.62 \\
35 \%\end{array}$ & $\begin{array}{l}2.95 \\
63 \%\end{array}$ \\
\hline 16 & $\begin{array}{l}\text { PCA- } \\
\text { RF }\end{array}$ & 10 & 0.9983 & $\begin{array}{l}9.83 \\
62\end{array}$ & $\begin{array}{l}15.6 \\
718\end{array}$ & $\begin{array}{l}3.86 \\
12 \%\end{array}$ & $\begin{array}{l}5.86 \\
53 \%\end{array}$ \\
\hline 16 & $\begin{array}{l}\text { DWT } \\
\text {-RF }\end{array}$ & 10 & 0.9997 & $\begin{array}{l}6.63 \\
62\end{array}$ & $\begin{array}{l}9.26 \\
91\end{array}$ & $\begin{array}{l}1.89 \\
7 \%\end{array}$ & $\begin{array}{l}2.52 \\
19 \%\end{array}$ \\
\hline 17 & $\begin{array}{l}\text { PCA- } \\
\text { RF }\end{array}$ & 10 & 0.998 & $\begin{array}{l}0.00 \\
1\end{array}$ & $\begin{array}{l}0.00 \\
14\end{array}$ & $\begin{array}{l}5.17 \\
09 \%\end{array}$ & $\begin{array}{l}6.36 \\
65 \%\end{array}$ \\
\hline 17 & $\begin{array}{l}\text { DWT } \\
\text {-RF }\end{array}$ & 10 & 0.999 & $\begin{array}{l}0.00 \\
1\end{array}$ & $\begin{array}{l}0.00 \\
12\end{array}$ & $\begin{array}{l}4.89 \\
99 \%\end{array}$ & $\begin{array}{l}6.16 \\
65 \%\end{array}$ \\
\hline 18 & $\begin{array}{l}\text { PCA- } \\
\text { RF }\end{array}$ & 10 & 0.9981 & $\begin{array}{l}0.00 \\
11\end{array}$ & $\begin{array}{l}0.00 \\
15\end{array}$ & $\begin{array}{l}5.14 \\
48 \%\end{array}$ & $\begin{array}{l}6.23 \\
19 \%\end{array}$ \\
\hline 18 & $\begin{array}{l}\text { DWT } \\
\text {-RF }\end{array}$ & 10 & 0.9988 & $\begin{array}{l}0.00 \\
12\end{array}$ & $\begin{array}{l}0.00 \\
18\end{array}$ & $\begin{array}{l}5.12 \\
23 \%\end{array}$ & $\begin{array}{l}6.56 \\
9 \%\end{array}$ \\
\hline 19 & $\begin{array}{l}\text { PCA- } \\
\text { RF }\end{array}$ & 10 & 0.9984 & $\begin{array}{l}8.90 \\
9\end{array}$ & $\begin{array}{l}15.5 \\
156\end{array}$ & $\begin{array}{l}3.50 \\
56 \%\end{array}$ & $\begin{array}{l}5.71 \\
77 \%\end{array}$ \\
\hline 19 & $\begin{array}{l}\text { DWT } \\
\text {-RF }\end{array}$ & 10 & 0.9987 & $\begin{array}{l}8.75 \\
6\end{array}$ & $\begin{array}{l}15.4 \\
582\end{array}$ & $\begin{array}{l}3.45 \\
89 \%\end{array}$ & $\begin{array}{l}5.63 \\
59 \%\end{array}$ \\
\hline 20 & $\begin{array}{l}\text { PCA- } \\
\text { RF }\end{array}$ & 10 & 0.9982 & $\begin{array}{l}0.00 \\
11\end{array}$ & $\begin{array}{l}0.00 \\
15\end{array}$ & $\begin{array}{l}5.01 \\
24 \%\end{array}$ & $\begin{array}{l}6.12 \\
81 \%\end{array}$ \\
\hline 20 & $\begin{array}{l}\text { DWT } \\
\text {-RF }\end{array}$ & 10 & 0.9973 & $\begin{array}{l}0.00 \\
19\end{array}$ & $\begin{array}{l}0.00 \\
14\end{array}$ & $\begin{array}{l}5.23 \\
56 \%\end{array}$ & $\begin{array}{l}6.45 \\
23 \%\end{array}$ \\
\hline 21 & $\begin{array}{l}\text { PCA- } \\
\text { RF }\end{array}$ & 10 & 0.9981 & $\begin{array}{l}10.0 \\
926\end{array}$ & $\begin{array}{l}16.8 \\
603\end{array}$ & $\begin{array}{l}3.98 \\
15 \%\end{array}$ & $\begin{array}{l}6.10 \\
96 \%\end{array}$ \\
\hline 21 & $\begin{array}{l}\text { DWT } \\
\text {-RF }\end{array}$ & 10 & 0.9986 & $\begin{array}{l}8.70 \\
6\end{array}$ & $\begin{array}{l}14.4 \\
13\end{array}$ & $\begin{array}{l}3.43 \\
45 \%\end{array}$ & $\begin{array}{l}5.22 \\
28 \%\end{array}$ \\
\hline 22 & $\begin{array}{l}\text { PCA- } \\
\text { RF }\end{array}$ & 10 & 0.9979 & $\begin{array}{l}0.00 \\
12\end{array}$ & $\begin{array}{l}0.00 \\
16\end{array}$ & $\begin{array}{l}5.46 \\
95 \%\end{array}$ & $\begin{array}{l}6.56 \\
6 \%\end{array}$ \\
\hline 22 & $\begin{array}{l}\text { DWT } \\
\text {-RF }\end{array}$ & 10 & 0.9994 & $\begin{array}{l}0.00 \\
06\end{array}$ & $\begin{array}{l}0.00 \\
08\end{array}$ & $\begin{array}{l}2.90 \\
49 \%\end{array}$ & $\begin{array}{l}3.36 \\
04 \%\end{array}$ \\
\hline 23 & $\begin{array}{l}\text { PCA- } \\
\text { RF }\end{array}$ & 10 & 0.9983 & $\begin{array}{l}9.65 \\
45\end{array}$ & $\begin{array}{l}16.5 \\
444\end{array}$ & $\begin{array}{l}3.81 \\
24 \%\end{array}$ & $\begin{array}{l}5.87 \\
58 \%\end{array}$ \\
\hline 23 & $\begin{array}{l}\text { DWT } \\
\text {-RF }\end{array}$ & 10 & 0.9987 & $\begin{array}{l}8.60 \\
74\end{array}$ & $\begin{array}{l}14.4 \\
555\end{array}$ & $\begin{array}{l}3.39 \\
89 \%\end{array}$ & $\begin{array}{l}5.13 \\
39 \%\end{array}$ \\
\hline 24 & $\begin{array}{l}\text { PCA- } \\
\text { RF }\end{array}$ & 10 & 0.9991 & $\begin{array}{l}0.00 \\
08\end{array}$ & $\begin{array}{l}0.00 \\
11\end{array}$ & $\begin{array}{l}3.53 \\
78 \%\end{array}$ & $\begin{array}{l}4.25 \\
5 \%\end{array}$ \\
\hline 24 & $\begin{array}{l}\text { DWT } \\
\text {-RF }\end{array}$ & 10 & 0.9976 & $\begin{array}{l}0.00 \\
12\end{array}$ & $\begin{array}{l}0.00 \\
17\end{array}$ & $\begin{array}{l}5.71 \\
04 \%\end{array}$ & $\begin{array}{l}6.94 \\
42 \%\end{array}$ \\
\hline 25 & $\begin{array}{l}\text { PCA- } \\
\text { RF }\end{array}$ & 10 & 0.9994 & $\begin{array}{l}7.88 \\
4\end{array}$ & $\begin{array}{l}12.2 \\
898\end{array}$ & $\begin{array}{l}2.25 \\
38 \%\end{array}$ & $\begin{array}{l}3.34 \\
37 \%\end{array}$ \\
\hline 25 & $\begin{array}{l}\text { DWT } \\
\text {-RF }\end{array}$ & 10 & 0.9994 & $\begin{array}{l}0.00 \\
07\end{array}$ & $\begin{array}{l}0.00 \\
09\end{array}$ & $\begin{array}{l}2.99 \\
05 \%\end{array}$ & $\begin{array}{l}3.60 \\
62 \%\end{array}$ \\
\hline
\end{tabular}




\section{FUTURE SCOPE}

(1) It can be used to detect poacher persons in forests to save the life of extinct species. Wildlife crime is one of the most profit giving illegal business worldwide. Nowadays, most of the precautionary measures that are being taken to reduce it are lacking effectivity and failing to prevent declining population of many endangered species, paving the way for the need of an innovative antipoaching solutions.

(2) To alarm farmers about the human or animal intrusion in the farm house which may cause harm to their crops. In many of the developing countries, agriculture is one of the rudimental occupational place of common people. Agriculture needs different kinds of technological advancements for improving crop yields. The intrusion of animals in the agricultural land or farmhouse and the theft of crops by human intruders cause heavy loss. In that case, this may help the farmers to be get warned before things gone far away from them.

(3) It can be used in banking sectors such as in ATM's or branches itself so that stealing of pin number, passwords of ATM or snatching of money from the customer by the crooks can be identified easily.

(4) It can also be used in jewellery shops and malls to detect the muggers.

(5) It can be used in the hostel and residential areas of academic institutions for the protection of students and staffs to detect and prevent unwanted intrusion of suspicious persons.

(6) It can also be used in the perspective of administration and to maintain law \& order by the Police, Army and other defence services for safety and well being of the society.

\section{CONCLUSION}

Here in this paper physical intruder detection system and what are the technological insiders within it have been explained. Understanding and visualizing its capability and scope it can said that it may be very much helpful for the people or society who wants to detect and prevent from unwanted peoples trespassing to their property, area, locality, forests etc. And in this paper it have been also attempted to place a mirror to reflect what works had been done in this domain in previous. Hope this will be helpful for all who wants to advance in this field and also wants to cope with the intruders .

\section{REFERENCES}

[1] Ramanan D, Sminchisescu C (2006) Training deformable models for localization. In: IEEE computer society conference on computer vision and pattern recognition, pp 206-21.

[2] Souto H, Musse SR (2011) Automatic detection of 2D human postures based on single images. In: Sibgrapi conference on graphics,patterns and images, pp 48-55.

[3] Babu A, Dube K, Mukhopadhyay S, Ghayvat H, Jithin KMV (2016) Accelerometer based human activities and posture recognition. In: International conference on data mining and advanced computing, pp367-373

[4] Miranda L, Vieira T, Martínez D, Lewiner T, Vieira AW, Campos MFM (2014) Online gesture recognition from pose kernel learning and decision forests. Pattern Recognit Lett 39(1):65-73.

[5] Agarwal A, Triggs B (2004) 3D human pose from silhouettes by relevance vector regression. In: Proc CVPR, vol 2, ppII-882-II-888.

[6] Zainordin FD, Lee HY, Sani NA, Yong MW, Chan CS (2012) Human pose recognition using kinect and rulebased system. In:World automation congress, pp 1-6

[7] MatthewA. Turk and Alex P. Pentland. 1991. Face recognition using eigenfaces. In Proceedings IEEE Computer Society Conference on Computer Vision and Pattern Recognition (CVPR'91). IEEE, 586-591.

[8] John G. Daugman. 1993. High confidence visual recognition of persons by a test of statistical independence. IEEETransactions on Pattern Analysis and Machine Intelligence 15, 11, 1148-1161.

[9] Anil K. Jain, LinHong, Sharath Pankanti, and Ruud Bolle. 1997. An identity-authentication system using fingerprints.Proceedings of the IEEE 85, 9, 1365-1388.

[10] A. K. Jain and N. Duta. 1999. Deformable matching of hand shapes for verification. In Proceedings of International Conference on Image Processing. 857861.

[11] Lily Lee and W. Eric L. Grimson. 2002. Gait analysis for recognition and classification. In Proceedings 5th IEEE International Conference on Automatic Face and Gesture Recognition, 2002. IEEE, 155-162.

[12] Jang Hee Yoo, Doosung Hwang, Ki Young Moon, and Mark S. Nixon. 2008. Automated human recognition by gait using neural network. In 1st Workshops on Image Processing Theory, Tools and Applications, IPTA 2008. $1-6$.

[13] Peter N. Belhumeur, Joao P. Hespanha, and David J. Kriegman. 1997. Eigenfaces vs. fisherfaces: Recognition using class specific linear projection. IEEE Transactions on Pattern Analysis and Machine Intelligence 19, 7, 711-720.

[14] Weili Ding , Bo Hu , Han Liu, Xinming Wang , Xiangsheng Huang3 . Human posture recognition based on multiple features and rule . Learning Received: 17 June 2019 / Accepted: 2 May 2020 / Published online: 2 June 2020 . International Journal of Machine Learning and Cybernetics (2020) 11:2529-2540

[15] Aravind Sundaresan, Amit RoyChowdhury, and Rama Chellappa. 2003. A hidden Markov model based framework for recognition of humans from gait sequences. In Proceedings 2003 International Conference on Image Processing, ICIP, Vol. 2. IEEE, II-93.

[16] Nikolaos V. Boulgouris and Zhiwei X. Chi. 2007. Human gait recognition based on matching of body components. Pattern Recognition 40, 6, 1763-1770.

[17] N. V. Boulgouris and Z. X. Chi. 2007. Gait recognition using radon transform and linear discriminant analysis. IEEE Transactions on Image Processing A Publication of the IEEE Signal Processing Society 16, 3, 731-740.

[18] Han Su and Fenggang Huang. 2006. Gait recognition using principal curves and neural networks. In 
International Symposium on Neural Networks. 238243.

[19] Springer Shmuel and Yogev Seligmann Galit. 2016. Validity of the kinect for gait assessment: A focused review.Sensors 16, 2, 194.

[20] Nikolaos V. Boulgouris and Zhiwei X. Chi. 2007. Human gait recognition based on matching of body components. Pattern Recognition 40, 6, 1763-1770.

[21] Qiong Cheng, Bo Fu, and Hui Chen. 2009. Gait recognition based on PCA and LDA. In International Symposium on Computer Science and Computational Technology (ISCSCI'09). Academy Publisher, 124127.

[22] Xuelong Li, Stephen J. Maybank, Shuicheng Yan, Dacheng Tao, and Dong Xu. 2008. Gait components and their application to gender recognition. IEEE Transactions on Systems, Man, and Cybernetics, Part C (Applications and Reviews) 38, 2, 145-155.

[23] Jiwen Lu and Erhu Zhang. 2007. Gait recognition for human identification based on ICA and fuzzy SVM through multiple views fusion. Pattern Recognition Letters 28, 16, 2401-2411.

[24] Claudia Nickel, Tobias Wirtl, and Christoph Busch. 2012. Authentication of smartphone users based on the way they walk using k-NN algorithm. In 8th International Conference on Intelligent Information Hiding and Multimedia Signal Processing. 16-20.

[25] Chiraz BenAbdelkader, Ross Cutler, Harsh Nanda, and Larry Davis. 2001. Eigengait: Motion-based recognition of people using image self-similarity. In Audio-and Video-Based Biometric Person Authentication. Springer, 284-294.

[26] Marcin Derlatka and Mikhail Ihnatouski. 2010. Decision tree approach to rules extraction for human gait analysis. In International Conference on Artificial Intelligence and Soft Computing. 597-604.

[27] Hoppe T, Kiltz S, Dittmann J. Security Threats to Automotive CAN Networks-Practical Examples and Selected Short-Term Countermeasures. Proceedings of the 27th International Conference SAFECOMP 2008.

[28] Amit Kale, Aravind Sundaresan, A. N. Rajagopalan, Naresh P. Cuntoor, Amit K. Roy-Chowdhury, Volker Kruger, and Rama Chellappa. 2004. Identification of humans using gait. IEEE Transactions on Image Processing 13, 9, 1163-1173.

[29] [29] Anil K. Jain, Arun Ross, and Sharath Pankanti. 2006. Biometrics: a tool for information security. IEEE Transactions on Information Forensics and Security 1, $2,125-143$.

[30] Anil Jain, Ruud Bolle, and Sharath Pankanti. 2006. Biometrics: Personal Identification in Networked Society, Volume 479. Springer Science \& Business Media.

[31] Ruud M. Bolle, Jonathan Connell, Sharath Pankanti, Nalini K. Ratha, andAndrewW. Senior. 2013. Guide to Biometrics. Springer Science \& Business Media.
[32] M. P. Murray. 1967. Gait as a total pattern of movement: Including a bibliography on gait. American Journal of Physical Medicine \& Rehabilitation 46, 1, 290-333.

[33] Ho TK (1995) Random decision forests. In: Proceedings of the $3^{\text {rd }}$ international conference on document analysis and recognition. Montreal, QC, pp 278-282

[34] Wang J, Huang Z, Zhang W, Patil A, Patil K, Zhu T, Shiroma EJ, Schepps MA, Harris TB (2017) Wearable sensor based human posture recognition. In: IEEE international conference on big data, pp 3432-3438.

[35] Lily Lee and W. Eric L. Grimson. 2002. Gait analysis for recognition and classification. In Proceedings 5th IEEE International Conference on Automatic Face and Gesture Recognition, 2002. IEEE, 155-162.

[36] Larson E, Nilsson, Dennis K, Jonsson E. An approach to specification-based attack detection for in-vehicle networks. IEEE Intelligent Vehicles Symposium 2008.

[37] Aravind Sundaresan, Amit RoyChowdhury, and Rama Chellappa. 2003. A hidden Markov model based framework for recognition of humans from gait sequences. In Proceedings 2003 International Conference on Image Processing, ICIP, Vol. 2. IEEE, II-93.

[38] Mark S. Nixon and John N. Carter. 2004. Advances in automatic gait recognition. In Proceedings 6th IEEE International Conference on Automatic Face and Gesture Recognition, 2004. IEEE, 139-144.

[39] Anil K. Jain, Arun Ross, and Salil Prabhakar. 2004. An introduction to biometric recognition. IEEE Transactions on Circuits and Systems for Video Technology 14, 1, 4-20.

[40] Gunnar Johansson. 1973. Visual perception of biological motion and a model for its analysis. Perception \& Psychophysics 14, 2, 201-211.

[41] Chen K, Wang Q (2016) Human posture recognition based on skeleton data. In: IEEE international conference on progress in informatics and computing, pp 618-622

[42] M. P. Murray, A. B. Drought, and R. C. Kory. 1964. Walking patterns of normal men. Journal of Bone \& Joint Surgery American Volume 46, 2, 335.

[43] Davrondzhon Gafurov. 2007. A survey of biometric gait recognition: Approaches, security and challenges. In Annual Norwegian Computer Science Conference, 19-21

[44] Peter N. Belhumeur, Joao P. Hespanha, and David J. Kriegman. 1997. Eigenfaces vs. fisherfaces: Recognition using class specific linear projection. IEEE Transactions on Pattern Analysis and Machine Intelligence 19, 7, 711-720.

[45] I. Haritaoglu, D. Harwood and L. S. Davis, "Ghost : A Human Body Part Labeling System Using Silhouettes," 14th International Conference on Pattern Recognition, 1998, Brisbane, Australia. 